\author{
Assistant Professor Engin TAS, PhD \\ E-mail: engintas@aku.edu.tr \\ Assistant Professor Ayca Hatice TURKAN, PhD \\ E-mail: aturkan@aku.edu.tr \\ Department of Statistics \\ Faculty of Science and Literature \\ Afyon Kocatepe University
}

\title{
REGULARIZED INDEX-TRACKING OPTIMAL PORTFOLIO SELECTION
}

\begin{abstract}
The aim of index-tracking approaches in portfolio optimization is to create a mimicking portfolio which tracks a specific market index. However, without regularization, this mimicking behavior of the index-tracking model is susceptible to the volatility in the market index and has negative effects on the tracking portfolio. We recast the index-tracking optimization problem by applying a form of regularization using the convex combination of $l_{1}$ and squared $l_{2}$ norm constraints on the portfolio weights. The proposed optimization model enables us to control the tracking performance and the sparsity structure of the portfolio simultaneously. A sample of assets from Borsa Istanbul (BIST) is used to demonstrate the performance of the regularized portfolios with various levels of regularization. Results indicated that the regularized portfolios obtained using this approach had better tracking performances with a desired sparsity structure compared to the standard index-tracking portfolio where no regularization is applied.
\end{abstract}

Keywords: Index-tracking, Portfolio optimization, Regularization, Portfolio management.

JEL Classification: C100, C610

\section{Introduction}

The foundations of the modern portfolio theory were laid by Harry Markowitz in 1950s. In the Markowitz's mean-variance portfolio theory, a meanvariance efficient portfolio is the one that has minimum variance for acceptable expected return or maximum expected return for acceptable variance among the possible portfolios. Allowing a unique efficiency frontier, Markowitz's model aims to minimize an objective function in order to obtain a mean-variance efficient portfolio where the variance is used as a risk measure (Jacobs et al., 2005). In the graph of the standard deviations of possible portfolios against the expected returns, any portfolio associated with a point on the efficient frontier curve is an optimum combination of assets which maximizes the return for a specific risk (standard 
deviation). The points in the area restricted by the curve have more risk for the same return or less return for the same risk. His work in portfolio theory provided him to win the Nobel Memorial Prize in Economic Sciences in 1990. Real world restrictions have led to the development of Markowitz mean-variance portfolio theory and proposal of new alternative models. Some of these models are applied by adding constraints such as buy-in threshold (quantity constraint), cardinality constraint and budget constraint (investment constraint), or by adding other additional constraints. DeMiguel et al. (2009) improved a strategy that includes constraints on the norm of the portfolio weight vector due to estimation errors resulting from sample means and covariances of the asset returns. They defined the 1-norm-constraint as $\|w\| \leq \delta$ and A-norm-constraint as $\left\|w^{\prime} A w\right\| \leq \delta$ where $\delta$ is a certain threshold, and they compared their norm-constrained approaches with the previous studies in the field and showed that there are certain correspondence between these studies.

Other studies tried to deal with the problems related to the computational complexity. Konno and Yamazaki (1991) developed the MAD (mean-absolute deviation) model using linear programming instead of quadratic programming since the standard deviation was replaced with the absolute deviation as a measure of dispersion. Based on linear programming, there are also other portfolio models such as MSAD (mean-semi absolute deviation) (Kamil and Ibrahim, 2005) and interval linear programming model (Şerban et al., 2015). Once again, in order to overcome the computational difficulties in estimating the covariance between each pair of assets when dealing with large amount of assets, Sharpe (1963) developed the single-index model based on a linear relationship between the return of an asset and a specific market index. Single-index models were followed by multi-index models, factor models and scenario models (Markowitz and Perold, 1981; Jacobs et al., 2005).

Index-tracking problem has also been studied in portfolio optimization. Many of these studies first transform the original optimization problem into the setting of the targeted optimization method and then try to solve the final optimization problem using the tools specific to that method. Some examples are heuristic (Beasley et al., 2003; Takeda et al., 2013) and metaheuristic (di Tollo and Maringer, 2009) approaches, mixed-integer linear programming (Guastaroba and Speranza, 2012), support vector machines (Karlow, 2013; Marcelino et al., 2015), hybrid genetic algorithms (Eddelbüttel, 1996), and multi-objective optimization (Li et al., 2011). However, there are not so many studies on regularization in indextracking problem. For instance, Brodie et al. (2009) proposed to add $l_{1}$ penalty to the objective function for partial index tracking. Giamouridis and Paterlini (2010) used Lasso and Ridge regression to apply $l_{1}$ or $l_{2}$ norms in order to regularize hedge fund clones. Takeda et al. (2013) developed an index tracking model based on $l_{0}$ and $l_{2}$ penalties. Yen and Yen (2014) applied a weighted $l_{1}$ and $l_{2}$ norms in mean-variance portfolio optimization problems using coordinate-wise descent. 


\section{Regularized Index-Tracking Optimal Portfolio Selection}

This paper follows a related, but different, approach to apply a convex combination of $l_{1}$ and $l_{2}$ norms on the index tracking problem.

\section{General framework}

We consider a portfolio of $n$ assets. Denote the return on asset $i$ by $r_{i}$, its mean by $\mu_{i}$ and its variance by $\sigma_{i}$. Let $\sigma_{i j}$ be the covariance between the returns of assets $i$ and $j$. The variance-covariance matrix of returns is denoted by $\Sigma$ which is widely used. The investment rate of asset $i$, that is, the weight of the asset is denoted by $w_{i}$. Denote the row vector containing the weights by $w^{T}=$ $\left(w_{1}, \ldots, w_{n}\right)$. The beta of an individual asset measuring systematic risk is denoted by $\beta_{i}=\frac{\sigma_{i M}}{\sigma_{m}^{2}}$ where $\sigma_{i M}$ is the covariance between the asset return and market index return and $\sigma_{M}^{2}$ is the variance of the market index return. The vector of individual asset betas is denoted by $\beta$.

In traditional index-tracking approach, it is desirable for the created portfolio to have as much return as the target market index (or target portfolio) while minimizing transaction costs. Tracking error, the variance of the difference between the portfolio return $\left(r_{P}\right)$ and the target market index return $\left(r_{M}\right)$, is defined as

$$
\begin{aligned}
\operatorname{Var}\left(r_{P}-r_{m}\right) & =\sigma_{P}^{2}+\sigma_{M}^{2}-2 \operatorname{Cov}\left(r_{P}, r_{M}\right) \\
& =\sigma_{P}^{2}+\sigma_{M}^{2}-2 \sigma_{M}^{2} \beta
\end{aligned}
$$

where $\sigma_{P}^{2}$ is the variance of the portfolio return. Index-tracking problem is to minimize $\sigma_{P}^{2}-\sigma_{M}^{2} \beta$ subject to $\mu^{t} \boldsymbol{w}=m$ and $\mathbf{1}^{T} \boldsymbol{w}=1$ since the index variance is independent of the portfolio weights.

\subsection{Regularized index-tracking optimization model}

Index-tracking portfolios without regularization have a strict mimicking behavior of the target market index. This makes it susceptible to the volatility in the market index and has negative effects on the tracking portfolio. In order to overcome this problem, a form of regularization has to be applied on the portfolio weights. There are 3 types of norm constraints in general, $l_{0}, l_{1}$ and $l_{2}$, used in previous studies in various settings. $l_{0}$ norm mitigates these negative effects by restricting the number of assets in the portfolio, but it involves combinatorial optimization and can become mathematically intractable when $n$ is large. On the other hand, $l_{1}$ norm constraint is a convex function of $\boldsymbol{w}$ and can also preserve sparsity in the portfolio. Furthermore, $l_{2}$ norm helps to avoid extreme portfolio weights by penalizing the size of the portfolio weights. Therefore, in order to benefit from the advantages of both norm constraints simultaneously, we propose to regularize the standard index-tracking problem by applying a convex 


\section{Engin Tas, Ayca Hatice Turkan}

combination of the $l_{1}$ and squared $l_{2}$ norm constraints on the portfolio weights. The new optimization problem becomes as

$$
\begin{array}{cc}
\underset{\mathbf{w}}{\operatorname{minimize}} & \mathbf{w}^{T} \Sigma \mathbf{w}-2 \sigma_{M}^{2} \beta^{T} \mathbf{w}+C\left(\alpha\|\mathbf{w}\|_{l_{1}}+(1-\alpha)\|\mathbf{w}\|_{l_{2}}^{2}\right), \\
\text { subject to } & \mu^{T} \mathbf{w}=m \\
& \mathbf{1}^{T} \mathbf{w}=1 .
\end{array}
$$

$C \in R^{+}$is a regularization parameter which controls the trade-off between the variance of the tracking error and the effect of the penalties on the portfolio weights. $\alpha \in[0,1]$ is the weight parameter which adjusts the relative weight of the $l_{1}$ and squared $l_{2}$ norms. When $\alpha=0$, the portfolio weights are regularized by the squared $l_{2}$ norm $\|\mathbf{w}\|_{l_{2}}^{2}$ only, when $0<\alpha<1, l_{1}$ norm $\|\mathbf{w}\|_{l_{1}}$ constraint is applied with the regularized covariance matrix $\Sigma^{R}=\Sigma+C I_{n \times n}$, and when $\alpha=1$, the portfolio weights are regularized by the $l_{1}$ norm $\|\mathbf{w}\|_{l_{1}}$ only. The constraint $\mu^{T} \mathbf{w}=$ $m$ is the target mean return constraint with the target mean return $m$, and $\mathbf{1}^{T} \boldsymbol{w}=1$ is the endowment constraint.

The Lagrangian corresponding to the optimization problem (1) is

$$
\begin{aligned}
L(\mathbf{w}, \lambda, \gamma ; \Sigma, C, \alpha)= & \mathbf{w}^{T} \Sigma \mathbf{w}-2 \sigma_{M}^{2} \beta^{T} \mathbf{w}+ \\
& C \alpha\|\mathbf{w}\|_{l_{1}}+C(1-\alpha)\|\mathbf{w}\|_{l_{2}}^{2}- \\
& \lambda\left(\mu^{T} \mathbf{w}-m\right)-\gamma\left(\mathbf{1}^{T} \mathbf{w}-1\right) \\
= & \mathbf{w}^{T} \Sigma \mathbf{w}+\sum_{i=1}^{n}\left(-2 \sigma_{M}^{2} \beta_{i} w_{i}+C \alpha\left|w_{i}\right|+\right. \\
& \left.C(1-\alpha) w_{i}^{2}-\gamma w_{i}-\lambda \mu_{i} w_{i}\right)+\lambda m+\gamma .
\end{aligned}
$$

The KKT conditions for the Lagrangian (2) are

$$
\begin{aligned}
2 w_{i} \sigma_{i}^{2}+2 \sum_{j \neq i}^{n} w_{j} \sigma_{i j}+2 C(1-\alpha) w_{i}-\gamma-\lambda \mu_{i} & =2 \sigma_{M}^{2} \beta_{i}-C \alpha \text { if } w_{i}>0, \\
2 w_{i} \sigma_{i}^{2}+2 \sum_{j \neq i}^{n} w_{j} \sigma_{i j}+2 C(1-\alpha) w_{i}-\gamma-\lambda \mu_{i} & =2 \sigma_{M}^{2} \beta_{i}+C \alpha \text { if } w_{i}<0, \\
\left|2 \sum_{j \neq i}^{n} w_{j} \sigma_{i j}-\gamma-\lambda \mu_{i}\right| \leq 2 \sigma_{M}^{2} \beta_{i}+C \alpha \text { if } w_{i}=0, & \mu^{T} \mathbf{w}=m, \\
\mathbf{1}^{T} \mathbf{w} & =1 .
\end{aligned}
$$

From the KKT conditions (3), it can be shown that when $w_{i} \neq 0$, at the stationary point 
Regularized Index-Tracking Optimal Portfolio Selection

$$
\begin{gathered}
w_{i}=\frac{\gamma+\lambda \mu_{i}-z_{i}+2 \sigma_{M}^{2} \beta_{i}-C \alpha}{2\left(\sigma_{i}^{2}+C(1-\alpha)\right)}, \text { if } w_{i}>0, \\
w_{i}=\frac{\gamma+\lambda \mu_{i}-z_{i}+2 \sigma_{M}^{2} \beta_{i}+C \alpha}{2\left(\sigma_{i}^{2}+C(1-\alpha)\right)}, \text { if } w_{i}<0,
\end{gathered}
$$

where $z_{i}=2 \sum_{j \neq i}^{n} w_{j} \sigma_{i j}$. Let $S_{+}=i: w_{i}>0$ and $S_{-}=i: w_{i}<0$, then we know that

$$
\begin{aligned}
\mathbf{1}^{T} \mathbf{w}= & \gamma\left(\sum_{i \in S_{+} \cup S_{-}} \frac{1}{2\left(\sigma_{i}^{2}+C(1-\alpha)\right)}\right)+\sum_{i \in S_{+} \cup S_{-}} \frac{\lambda \mu_{i}-z_{i}+2 \sigma_{M}^{2} \beta_{i}}{2\left(\sigma_{i}^{2}+C(1-\alpha)\right)}+ \\
& C \alpha\left(\sum_{i \in S_{-}} \frac{1}{2\left(\sigma_{i}^{2}+C(1-\alpha)\right)}-\sum_{i \in S_{+}} \frac{1}{2\left(\sigma_{i}^{2}+C(1-\alpha)\right)}\right) \\
\mu^{T} \mathbf{w}= & \gamma\left(\sum_{i \in S_{+} \cup S_{-}} \frac{\mu_{i}}{2\left(\sigma_{i}^{2}+C(1-\alpha)\right)}\right)+\sum_{i \in S_{+} \cup S_{-}} \frac{\lambda \mu_{i}^{2}-\mu_{i} z_{i}+2 \sigma_{M}^{2} \mu_{i} \beta_{i}}{2\left(\sigma_{i}^{2}+C(1-\alpha)\right)}+ \\
& C \alpha\left(\sum_{i \in S_{-}} \frac{\mu_{i}}{2\left(\sigma_{i}^{2}+C(1-\alpha)\right)}-\sum_{i \in S_{+}} \frac{\mu_{i}}{2\left(\sigma_{i}^{2}+C(1-\alpha)\right)}\right) .
\end{aligned}
$$

For a given $\lambda$, using $\boldsymbol{w}^{T} \mathbf{1}=1, \gamma$ can be found as

$$
\gamma=\frac{1-\sum_{i \in S_{+} \cup S_{-}} \frac{\lambda \mu_{i}-z_{i}+2 \sigma_{M}^{2} \beta_{i}}{2\left(\sigma_{i}^{2}+C(1-\alpha)\right)} C \alpha\left(\sum_{i \in S_{-}} \frac{1}{2\left(\sigma_{i}^{2}+C(1-\alpha)\right)}-\sum_{i \in S_{+}} \frac{1}{2\left(\sigma_{i}^{2}+C(1-\alpha)\right)}\right)}{\left(\sum_{i \in S_{+} \cup S_{-}} \frac{1}{2\left(\sigma_{i}^{2}+C(1-\alpha)\right)}\right)}
$$

Similarly, by $\boldsymbol{w}^{T} \boldsymbol{\mu}=m$, for a given $\gamma$, we have

$$
\lambda=\frac{m-\sum_{i \in S_{+} \cup S_{-}} \frac{\mu_{i}\left(\gamma-z_{i}+2 \sigma_{M}^{2} \beta_{i}\right)}{2\left(\sigma_{i}^{2}+C(1-\alpha)\right)}-C \alpha\left(\sum_{i \in S_{-}} \frac{\mu_{i}}{2\left(\sigma_{i}^{2}+C(1-\alpha)\right)}-\sum_{i \in S_{+}} \frac{\mu_{i}}{2\left(\sigma_{i}^{2}+C(1-\alpha)\right)}\right)}{\left(\sum_{i \in S_{+} \cup S_{-}} \frac{\mu_{i}^{2}}{2\left(\sigma_{i}^{2}+C(1-\alpha)\right)}\right)}
$$

We followed Yen and Yen (2014) to adapt the coordinate-wise gradient descent algorithm to solve the regularized index-tracking problem iteratively. Assuming that $\lambda$ and $\gamma$ are fixed, each $w_{i}$ is updated according to the following formula

$$
w_{i} \leftarrow \frac{S T\left(\gamma+\lambda \mu_{i}-z_{i}+2 \sigma_{M}^{2} \beta_{i}, C \alpha\right)}{2\left(\sigma_{i}^{2}+C(1-\alpha)\right)}
$$

DOI: 10.24818/18423264/52.3.18.09 
where $S T(x, y)=\operatorname{sign}(x) \max (|x|-y, 0)$. Weights are initialized with $w_{1}=$ $w_{2}=\cdots=w_{n}=n^{-1}$, and $\gamma$ and $\lambda$ are chosen to satisfy $\gamma+\lambda \max _{i} \mu_{i}+$ $2 \sigma_{M}^{2} \max _{i} \beta_{i}>C$. The weights, $\gamma$ and $\lambda$ are updated sequentially until $\mid w_{k+1}-$ $w_{k} \mid<\epsilon$.

\section{Empirical results}

Let us now consider constructing a portfolio consisting of 18 assets. For each asset, we used the monthly price data from Borsa Istanbul (BIST) for the period starting from January 2009 until June 2013. These assets were selected among the most 20 active assets. Two of the assets were not used due to lack of data, therefore 18 assets were included in the study. BIST 100 index was used as the target market index for tracking. We set the target mean return equal to the BIST-100 index mean return in the training period $(m=2.54)$. Table 1 provides the expected returns, standard deviations and betas for the selected assets.

Table 1. Statistical parameters of monthly asset returns

\begin{tabular}{lcll} 
Asset & $\mu$ & \multicolumn{1}{c}{$\sigma$} & \multicolumn{1}{c}{$\beta$} \\
\hline Akbank & 2.11 & 10.37 & -0.08 \\
Bim & 3.49 & 7.04 & 0.17 \\
Besiktas Futbol Yatirim & 4.57 & 25.58 & 0.87 \\
Eregli Demir Celik & 1.38 & 9.35 & 0.39 \\
Garanti & 2.93 & 11.49 & 0.04 \\
Halkbankasi & 3.33 & 13.06 & 0.05 \\
Is Bankasi (C) & 2.23 & 10.28 & 0.24 \\
Koc Holding & 3.54 & 11.16 & 0.16 \\
Kardemir (D) & 3.55 & 11.01 & 0.35 \\
Petkim & 2.54 & 9.77 & 0.46 \\
Sabanci Holding & 2.81 & 11.47 & 0.25 \\
Turkcell & 0.87 & 6.61 & 0.12 \\
Turk Hava Yollari & 5.29 & 12.55 & 0.42 \\
Tupras & 3.12 & 9.19 & 0.08 \\
Vakifbank & 3.50 & 13.30 & 0.14 \\
Vestel & 2.63 & 13.68 & 0.37 \\
Yapi ve Kredi Bankasi & 2.05 & 11.81 & 0.08 \\
Zorlu Enerji & 1.83 & 14.79 & 0.75
\end{tabular}

First of all, the data were divided into two groups such as train and test. We used the first 42 months for training, and the last 12 months for testing. $C$ and $\alpha$ were selected from $\{0,1,10,100,100\}$ and $[0,1]$ respectively. For a given $C$ and $\alpha$, optimal portfolio was found using only the training period and the performance of the optimal portfolio was evaluated on the test period. We also performed another parameter search for $C$ and $\alpha$ in a higher precision, we created a 2-D grid 
for the parameters where $C \in[0,1000]$ and $\alpha \in[0,1]$ again, but with uniformly spaced values incremented by 0.1 . However, there were not any significant differences from the previous setting. Therefore, without loss of generalization, tracking performances of the regularized portfolios are summarized in Figure 1 for specific values of $C$ and $\alpha$. Cumulative monthly returns of the regularized portfolios are demonstrated with respect to the index returns. There is not any regularization on the portfolio when $C=0$, and this corresponds to the standard index-tracking portfolio. When $C>0$, a specific regularization is applied to the portfolio weights in the convex combination of $l_{1}$ and squared $l_{2}$ norm constraints weighted by the parameter $\alpha$. Results showed that, all portfolios are tracking the index successfully in the training period, however, portfolios with little or no regularization are began to deviate from the index significantly in the test period. Portfolios with moderate regularization have a more determined attitude which is much more desirable compared with the other portfolios. That is, portfolios with no or less regularization have more fluctuations in the test period of tracking. Thus, regularized portfolios are much more robust to the volatilities in the market index.

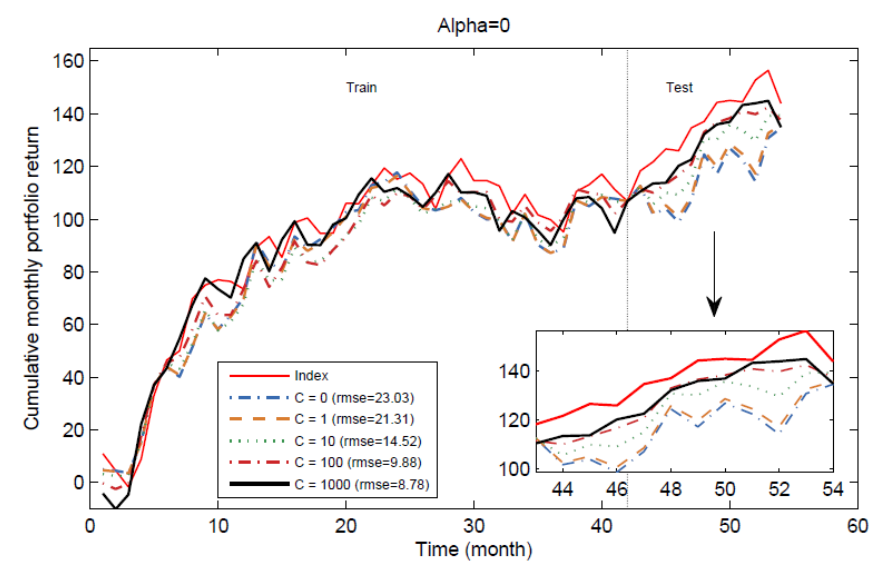

DOI: 10.24818/18423264/52.3.18.09 

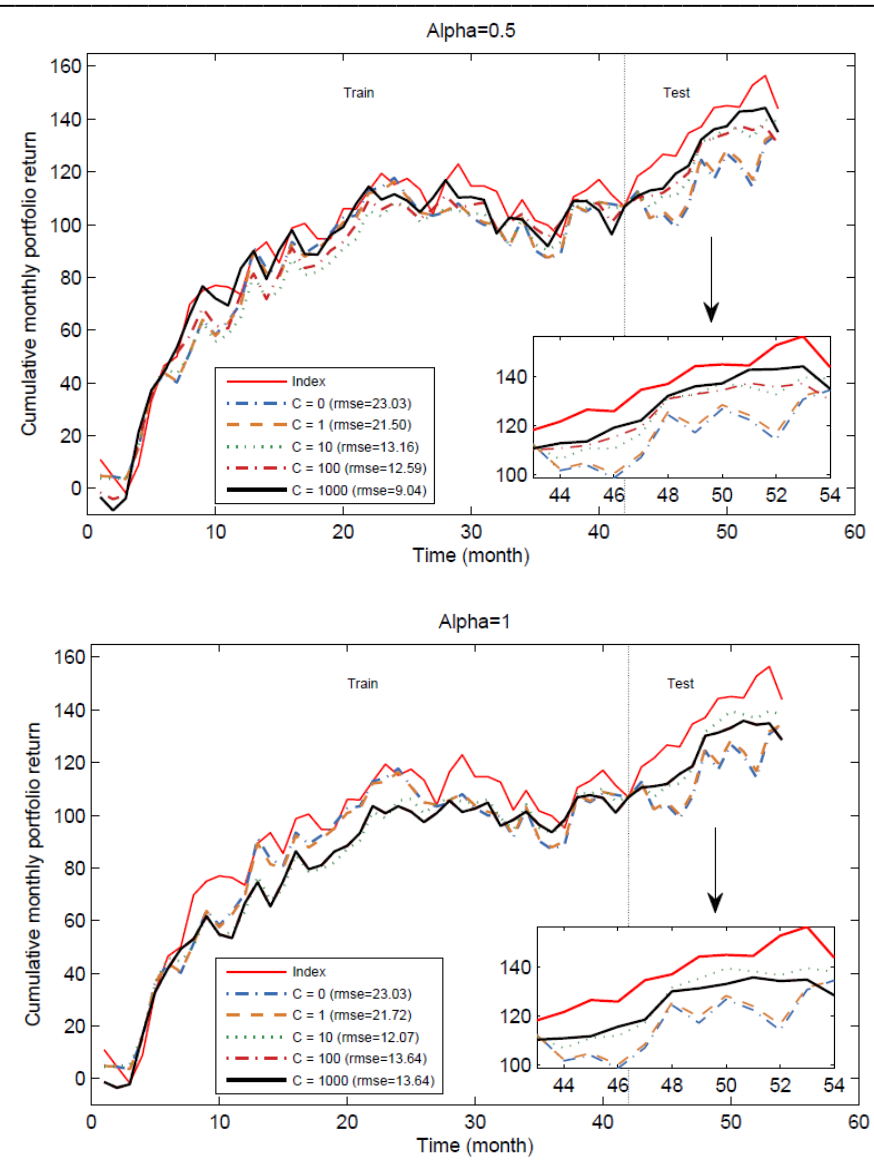

Figure 1. Cumulative monthly returns of regularized portfolios and the market index

Considering $\alpha$, root mean square error (rmse) between the cumulative portfolio and the index returns becomes a little larger as $\alpha$ increases. The changes in the portfolio weights are plotted against different levels of $(C, \alpha)$ in Figure 2. One can figure out how the convergence characteristics of the portfolio weights are differed with respect to different magnitudes of $C$ and different forms of regularization (specified by $\alpha$ ). When $\alpha=0$, portfolio weights are regularized by $l_{2}$ norm only, and proportion of active assets (PoA) always equal to 1, i.e., all assets are active, but proportion of short-sales (PoS) decreases as $C$ increases. There are no short-sales when $C$ becomes very large. In the case of $\alpha=1$, portfolio weights are regularized by $l_{1}$ norm only, and as $C$ increases up to a certain value, PoS decreases to 0 and PoA decreases to a certain small value. No changes are observed in PoA and PoS after this level of $C$. In other words, portfolio weights are stabilized at a specific level of $C$, the majority of the weights 


\section{Regularized Index-Tracking Optimal Portfolio Selection}

are converged to 0 and the number of active assets are limited to a relatively small number. This facilitates a certain sparsity structure in the portfolio.

For the case of $\alpha=0.5$, regularized portfolio tries to satisfy both objectives given in the above settings $(\alpha=0,1)$. PoA decreases up to a certain level, but then increases as $C$ gets much larger. On the other hand, as $C$ increases, PoS decreases to 0 at a certain level of $C$ and remains constant after this level. This choice of $\alpha$ plays a balancing role between the two settings.
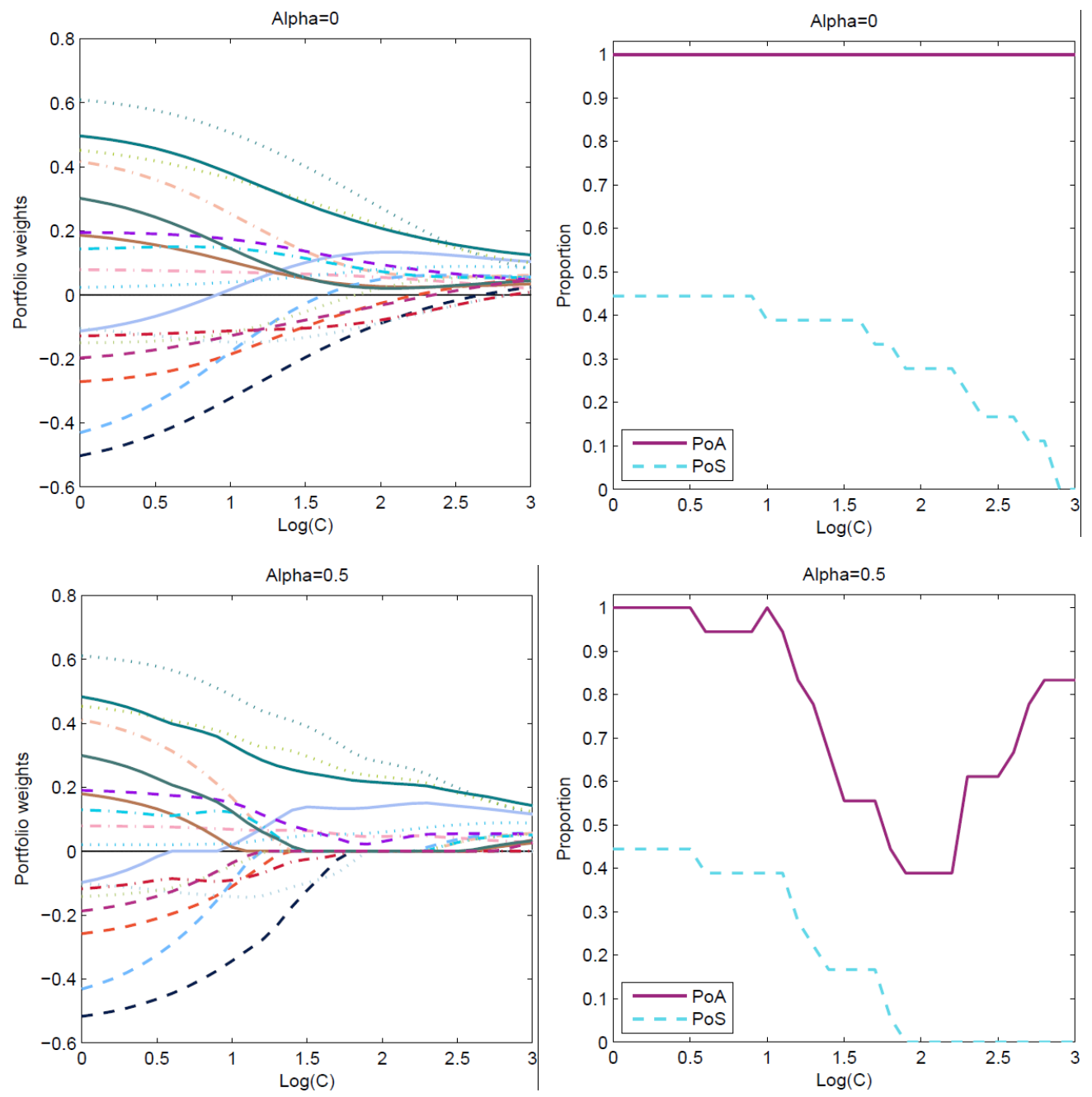

DOI: 10.24818/18423264/52.3.18.09 
Engin Tas, Ayca Hatice Turkan
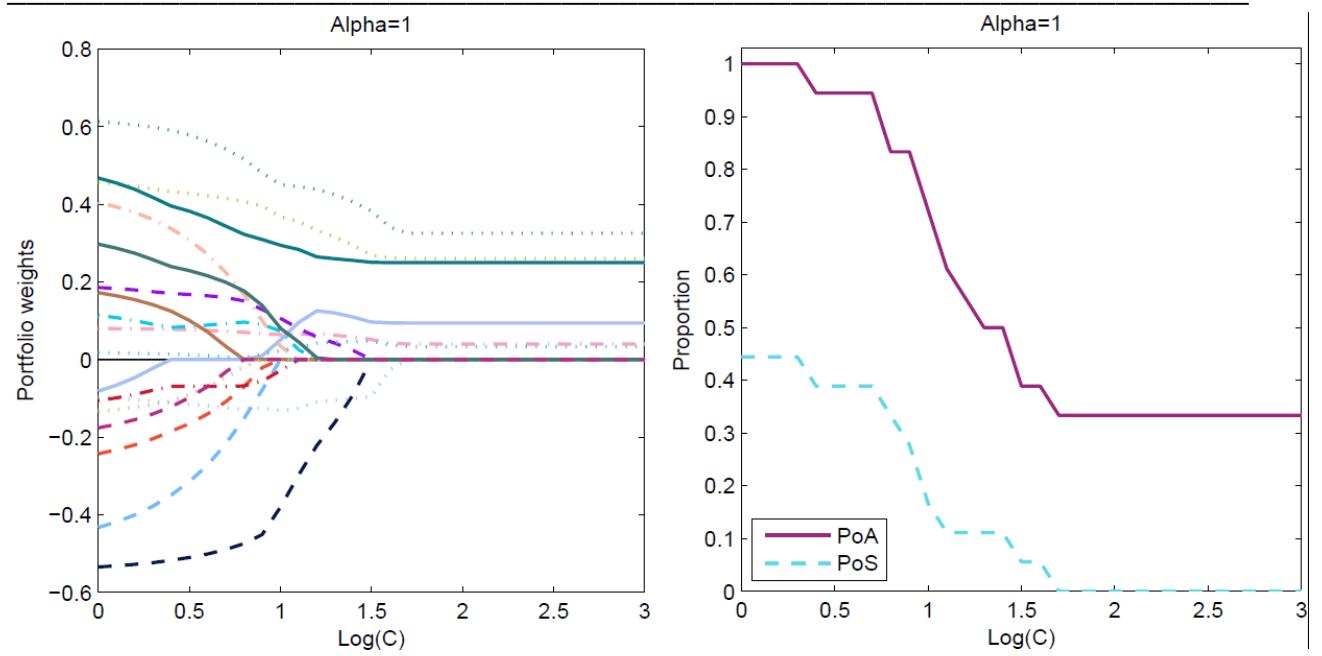

Figure 2. Portfolio weights, proportion of active assets (PoA) and proportion of short-sales (PoS) according to different levels of regularization

According to the Sharpe ratio, the best regularized portfolio is the one with $C=100$ and $\alpha=0$. All regularized portfolios with various $\alpha$ levels have smaller standard deviations of monthly returns than the standard portfolio has $(C=0)$. Standard deviation of monthly returns drops as $C$ gets larger at all levels of $\alpha$. When $C$ is around 100, it stabilizes and then increases a little as $C$ gets much larger $(C=1000)$.

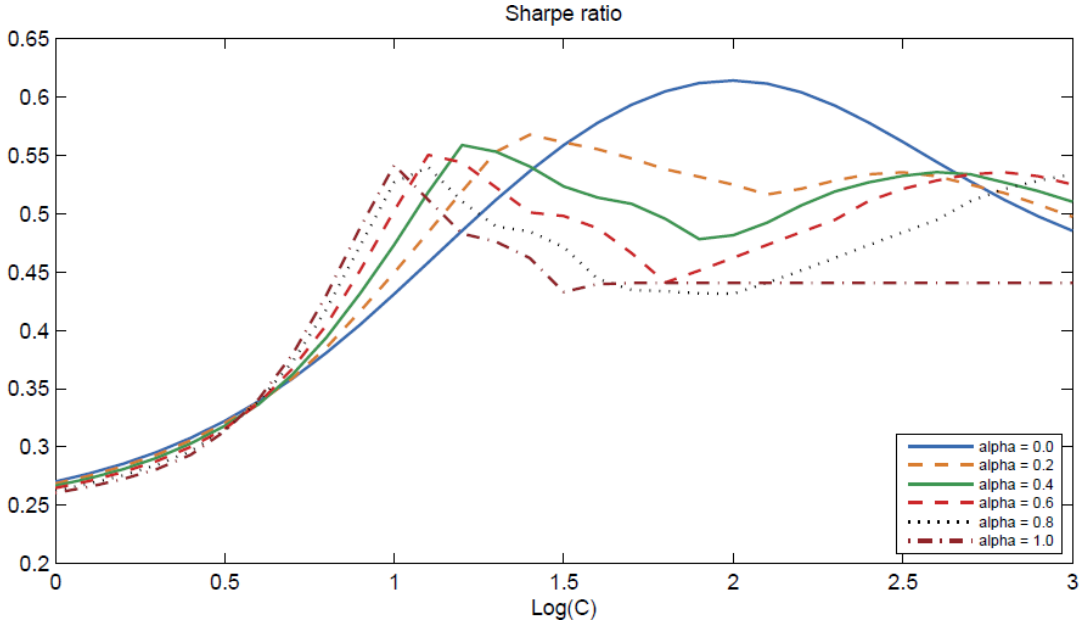




\section{Regularized Index-Tracking Optimal Portfolio Selection}

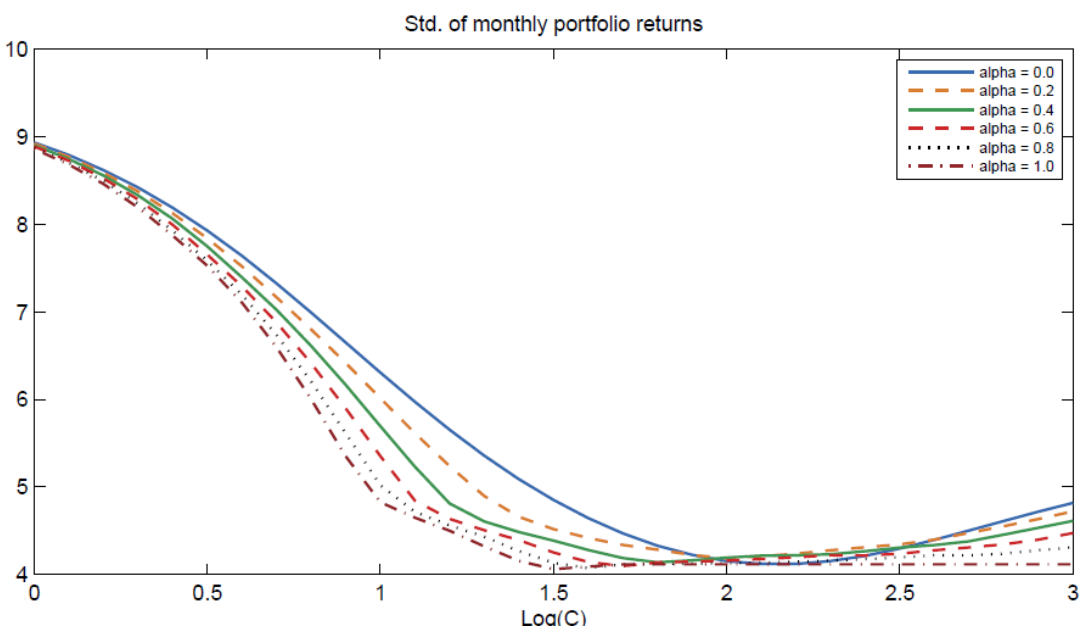

Figure 3. Sharpe ratio and std. of monthly portfolio returns according to different levels of regularization

\section{Conclusion}

Investors may have several goals in creating portfolios. An investor wants a portfolio that keeps track of a certain index with a specified return while others want a portfolio that minimizes transaction costs. Another investor wants to have both goals accomplished up to a certain level. Therefore, this paper proposes a well-defined flexible regularized optimization model for the index-tracking problem where one can control the tracking performance and the sparsity of the portfolio simultaneously by controlling the weight parameter $\alpha$. This provides a stabilizer between $l_{1}$ norm and squared $l_{2}$ norm penalties on the index-tracking problem.

We do not suggest any optimal regularization parameter $C$ and weight parameter $\alpha$ in this study since these parameters are data-dependent. However, the results indicated that, all regularized portfolios performed better than the standard index-tracking portfolio. Among the regularized portfolios, an investor can make his own decision by evaluating various performance criteria such as the tracking error, PoA, PoS, Sharpe ratio and etc. based on the goals of the target portfolio.

\section{REFERENCES}

[1] Beasley, J.E., Meade, N., Chang, T.J. (2003), An Evolutionary Heuristic for the Index Tracking Problem; European Journal of Operational Research, 148(3), 621643;

[2] Brodie, J., Daubechies, I., De Mol, C., Giannone, D., Loris, I. (2009), Sparse and Stable Markowitz Portfolios; Proceedings of the National Academy of Sciences, 106(30), 12267-12272;

DOI: 10.24818/18423264/52.3.18.09 
Engin Tas, Ayca Hatice Turkan

[3] DeMiguel, V., Garlappi, L., Nogales, F.J., Uppal, R. (2009), A Generalized Approach to Portfolio Optimization: Improving Performance by Constraining Portfolio Norms; Management Science, 55(5), 798-812;

[4] Eddelbüttel, D. (1996), A Hybrid Genetic Algorithm for Passive Management.

In: Working Paper presented at the Second conference on computing in economics and

Finance, Society of computational economics, Geneves, Suisse;

[5] Giamouridis, D., Paterlini, S. (2010), Regular(ized) Hedge Fund Clones;

Journal of Financial Research, 33(3), 223-247;

[6] Guastaroba, G., Speranza, M.G. (2012), Kernel Search: An Application to the Index Tracking Problem; European Journal of Operational Research, 217(1), 54-68; [7] Jacobs, B.I., Levy, K.N., Markowitz, H.M. (2005), Portfolio Optimization with Factors, Scenarios, and Realistic Short Positions; Operations Research, 53(4), 586599 ;

[8] Kamil, A.A., Ibrahim, K. (2005), The Downside Risk Optimal Portfolio Selection Problem. In: IRCMSA Proceedings;

[9] Karlow, D. (2013), Comparison and Development of Methods for Index

Tracking. PhD thesis, Frankfurt (Main), Frankfurt School of Finance \& Management, Diss., 2013;

[10] Konno, H., Yamazaki, H. (1991), Mean-Absolute Deviation Portfolio Optimization Model and its Applications to Tokyo Stock Market; Management science, 37(5), 519-531;

[11] Li, Q., Sun, L., Bao, L. (2011), Enhanced Index Tracking Based on MultiObjective Immune Algorithm; Expert Systems with Applications, 38(5), 6101-6106;

[12] Markowitz, H.M., Perold, A.F. (1981), Portfolio Analysis with Factors and Scenarios; The Journal of Finance, 36(4), 871-877;

[13] Marcelino, S., Henrique, P.A., Albuquerque, P.H.M. (2015), Portfolio

Selection with Support Vector Machines in Low Economic Perspectives in Emerging

Markets; Economic Computation \& Economic Cybernetics Studies \& Research, 49(4); ASE Publishing;

[14] Sharpe, W.F. (1963), A Simplified Model for Portfolio Analysis; Management science, 9(2), 277-293;

[15] Şerban, F., Costea, A., Ferrara, M. (2015), Portfolio Optimization Using Interval Analysis; Economic Computation and Economic Cybernetics Studies and Research, ASE Publishing, 48(1), 125-137;

[16] Takeda, A., Niranjan, M., Gotoh, J.Y., Kawahara, Y. (2013), Simultaneous Pursuit of Out-Of-Sample Performance and Sparsity In Index Tracking Portfolios; Computational Management Science, 10(1), 21-49;

[17] di Tollo, G., Maringer, D. (2009), Metaheuristics for the Index Tracking

Problem. In: Metaheuristics in the Service Industry, Springer, pp 127-154;

[18] Yen, Y.M., Yen, T.J. (2014), Solving Norm Constrained Portfolio Optimization via Coordinate-Wise Descent Algorithms; Computational Statistics \& Data Analysis, 76, 737-759. 